\section{Recurrencia de síndrome de Tako-Tsubo: un caso peruano}

\section{Recurrence of Tako-Tsubo syndrome. Case report}

\section{Sr. Editor:}

La cardiomiopatía de Tako-Tsubo (CT) fue descrita por Sato et al, en 1990, y reportada por primera vez en Japón, también llamada "síndrome de disfunción transitoria del ventrículo izquierdo" ya que produce una disfunción severa pero reversible y con buen pronóstico para el paciente ${ }^{1}$. Este síndrome afecta generalmente a mujeres de 50 a 80 años, con pocos factores de riesgo cardiovascular, y habitualmente está desencadenada por estrés emocional y/o físico debido al aumento de catecolaminas ${ }^{2,3}$. La CT tiene una presentación clínica similar al síndrome de infarto agudo del miocardio (SICA), por lo tanto, su diagnóstico es de suma importancia ya que representa el 1,7\% al 2,2\% de todos los casos diagnosticados como SICA ${ }^{1,4}$.

En el diagnóstico de CT se proponen cuatro criterios modificados por la Clínica Mayo, basados en la presentación clínica del paciente: 1) La discinesia o acinesia transitoria de los segmentos apicales y medios del ventrículo izquierdo, con distribución vascular correspondiente a más de una arteria epicárdica; 2) La ausencia de enfermedad coronaria obstructiva o evidencia de rotura aguda de placa en la angiografía; 3) La nueva aparición de anomalías en el trazado ECG, como elevación del segmento ST o inversión de la onda T; 4) Ausencia de traumatismo craneoencefálico reciente o hemorragia subaracnoidea, feocromocitoma, miocarditis, miocardiopatía hipertrófica o "aturdimiento miocárdico de origen isquémico"s.

Una paciente de sexo femenino, de 74 años, ingresó al Servicio de Emergencias por presentar un episodio de dolor retroesternal opresivo de 1 hora de evolución, posterior a una discusión familiar. El dolor, irradiado al miembro superior izquierdo y a la región posterior del tórax, estuvo asociado a diaforesis, palpitaciones, mareo y sensación de lipotimia. Antecedentes de hipertensión arterial, diabetes mellitus, dislipidemia y diagnóstico tres años antes de cardiomiopatía de Tako-Tsubo, con tratamiento ambulatorio con enalapril, metfor- mina, atorvastatina, aspirina. El examen físico al ingreso mostró PA: 130/90 mmHg, FC: 70 latidos/ min, Sat $\mathrm{O}_{2 .} 99 \%$, FR: 16 , temperatura de $36,8^{\circ} \mathrm{C}$. Ruidos cardíacos rítmicos, sin soplos; pulsos periféricos normales, sin ingurgitación yugular, ni crépitos en las bases pulmonares. Resto del examen físico sin alteraciones. Exámenes auxiliares de ingreso: leucocitos 6.500; abastonados $0 \%$; segmentados 69\%; Hb $11 \mathrm{~g} / \mathrm{dL}$; plaquetas 216000 ; glucosa $165 \mathrm{mg} / \mathrm{dL}$; urea $38 \mathrm{mg} / \mathrm{dL}$; creatinina 0,85 $\mathrm{mg} / \mathrm{dL}$. Perfil lipídico normal. Electrocardiograma de ingreso mostró ritmo sinusal con infra desnivel de ST en V4- V6, ondas T invertidas en V2- V6. Marcadores de daño miocárdico: troponinas 0,2 (VR: 0,1 ng/ml); CPK-MB 114 (VR: 7-25 U/L). Seis horas después se volvieron a medir: troponinas 1,65 y CPK-MB 108,8. Con estos resultados se diagnosticó un síndrome coronario agudo sin ST elevado (SICA STNE); se administraron aspirina, clopidogrel, atorvastatina, atenolol, isorbide. Al día siguiente se le realizó un ecocardiograma que mostró hipocinesia leve anterolateral y apical, con fracción de eyección (FEV) de aproximadamente $60 \%$.

Tres días después del ingreso se le realizó un cateterismo cardíaco, donde se visualizaron arterias coronarias normales (Figura 1) llegándose al diagnóstico de síndrome de Tako-Tsubo o cardiomiopatía de Tako-Tsubo.

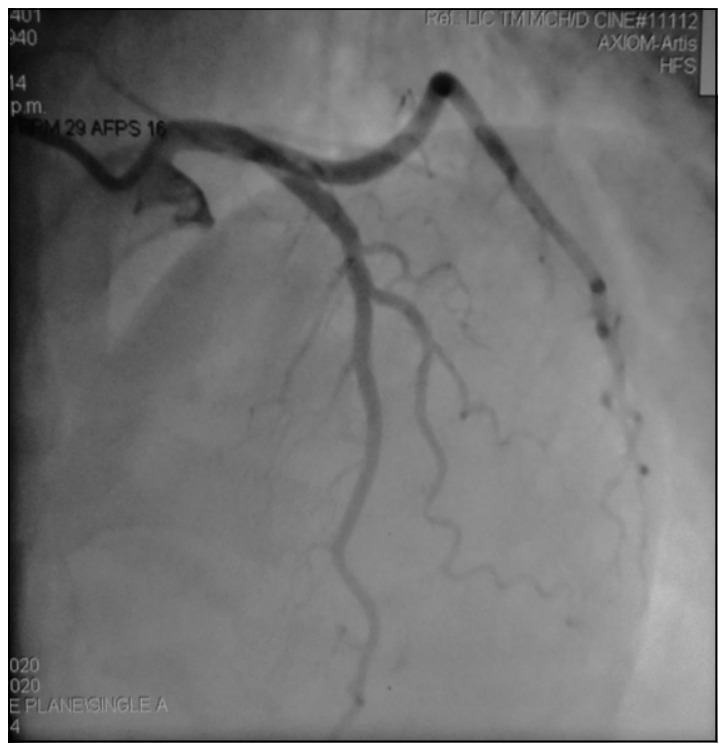

Figura 1. Coronario angiografia que muestra arterias coronarias sin oclusión. 
La paciente evolucionó favorablemente, por lo que al sexto día del ingreso fue dada de alta con indicación de enalapril, aspirina, clopidogrel, atorvastatina y metformina.

Esta paciente se presentó como un caso de infarto agudo del miocardio e inicialmente se le administró el respectivo tratamiento estándar. Sin embargo, para la confirmación de este diagnóstico se debió observar en el cateterismo una obstrucción de las arterias coronarias, lo cual no fue así permitiendo descartarlo. En cambio, se diagnosticó una cardiomiopatía de Tako-Tsubo, de acuerdo con los criterios de la Clínica Mayo ${ }^{5}$. Por otra parte, la paciente ya contaba con un episodio previo de CT, diagnosticado tres años antes en el Instituto del Corazón (INCOR). La recurrencia de CT es poco común ( $0 \%$ a $11,4 \%)$, lo que hace interesante el presente caso ${ }^{6}$.

Consideramos que la cardiomiopatía de Tako-Ttsubo puede ser incluida como un diagnóstico diferencial en mujeres mayores de 50 años, con antecedentes de estrés físico o emocional. La $\mathrm{CT}$, al tratarse de una alteración transitoria y no morfológica, tiene un pronóstico favorable en comparación con un infarto agudo del miocardio.

Zoraida del Solar-Moreno ${ }^{1,2, a}$, Humberto
Ulloa-Montoya ${ }^{1, b}$, Laura Orellana-Cuellar ${ }^{1,2, a}$
${ }^{1}$ Facultad de Medicina San Fernando.
Universidad Nacional Mayor de San Marcos.
Lima, Perú.
${ }^{2}$ Sociedad Científica de San Fernando.
Lima, Perú.
${ }^{a}$ Estudiante de Medicina.
${ }^{b}$ Médico Cardiólogo.

\section{Referencias}

1. Rojas Jiménez S, Valle L, Sebastián J. Cardiomiopatía de Takotsubo, el gran imitador del infarto agudo del miocardio. CES Med 2012; 26 (1): 107-20.

2. Pérez Pérez FM, Sánchez Salado J. Síndrome de Tako-Tsubo. Discinesia transitoria del ventrículo izquierdo. SEMERGEN-Med Fam 2014; 40 (2): 73-9.

3. Nascimento FO, Santana O, Pérez-Caminero M, Benjo AM. The characteristics of stress cardiomyopathy in an ethnically heterogeneous population. Clinics 2011; 66 (11): 1895-9.

4. Rau CM, Kauffmann M, Rau CL, Cereceda M, Castro G, Massardo T. Síndrome de Tako-Tsubo: caso clínico. Rev Med Chile 2011; 139 (3): 348-52.

5. Obón Azuara B, Ortas Nadal MR, Gutiérrez Cía I, Villanueva Anadón B. Cardiomiopatía de Takotsubo: disfunción transitoria apical de ventrículo izquierdo. Med Intensiva 2007; 31 (3): 146-52.

6. Rotondi F, Manganelli F, Capasso M, Lanzillo T, Bellizzi G, Sauro R. Recurrence of Tako-Tsubo syndrome, idiopathic dilated cardiomyopathy, and iterative ventricular tachycardia: just a fortuitous coincidence or a pathophysiological link? Eur Rev Med Pharmacol Sci 2013; 17 (22): 3117-20.

Conflictos de intereses: ninguno que declarar

Correspondencia a:

Zoraida del Solar Moreno

Av. Domínicos 253. Lima 13, Perú.

zori26dsm@hotmail.com 